\title{
Modelling of Size Effect with Regularised Continua
}

\begin{abstract}
H. Askes, A. Simone, L. J. Sluys
A nonlocal damage continuum and a viscoplastic damage continuum are used to model size effects. Three-point bending specimens are analysed, whereby a distinction is made between unnotched specimens, specimens with a constant notch and specimens with a proportionally scaled notch. Numerical finite element simulations have been performed for specimen sizes in a range of 1:64. Size effects are established in terms of nominal strength and compared to existing size effect models from the literature.
\end{abstract}

Keywords: size effect, modelling, statistic and deterministic size effect, stress fluctuation.

\section{Introduction}

When the dimensions of a structure are scaled proportionally (i.e. keeping the ratios between the relevant dimensions that have been fixed), the mechanical properties of the various samples differ. This phenomenon is known as size effect, see for instance $[1,2,3,4]$ and references therein. Being able to model size effects is relevant since the size range of specimens that can be tested experimentally is limited, and extrapolations towards very large specimen sizes must be made theoretically. It has sometimes been claimed that a proper modelling of size effects includes extensions towards the very large size range as well as the very small size range. However, in the very small size range the mesoscopic or microscopic constituents start to play a dominant role [5], and it must be questioned whether the traditional assumptions of continuum mechanics still hold.

Size effects have different origins. Originally, it was thought that merely a statistical size effect exists, which can be understood from the fact that larger specimens have a bigger chance that a weakest-link is present. More recently it has been recognised that also a deterministic size effect (also known as an energetic size effect) exists, which has been exemplified in tests where theoretically predicted stress gradients are dominant over local stress fluctuations (e.g. bending tests). Furthermore, different size effect trends are observed for unnotched specimens on, the one hand, and notched specimens where the notch scales proportionally, on the other.

In the past decades, many formulas have been proposed in order to capture size effects, most notably the Multi-Fractal Scaling Law (MFSL) by Carpinteri and co-workers and the Size Effect Law (SEL) by Bažant. These proposed models more or less coincide in the size range where experimental data are available; however, for the small-size limit and the large-size limit important differences may be present. To contribute additional arguments in this discussion, an attempt is made here to interpret size effects purely from a material modelling point of view.

Size effects occur when characteristic lengths at the structural level and at the material level interact - increasing the specimen size while using the same material implies that the material length scale remains constant. Classical continua in an elasticity, plasticity or damage context do not contain a material length scale. As a result, they are unable to describe size effects. In contrast, enhanced continua may be used in which an intrinsic length scale is incorporated as an additional material parameter. With these so-called nonlocal continuum descriptions, size-effects can be described as has been shown for instance in $[6,7,2,8,9,10]$. In $[11,12]$ the occurrence of size effects has been used to estimate the internal length scale, thus further establishing the relation that exists between the presence of an internal length scale and the occurrence of size effects. Finally, in [13] a nonlocal damage continuum is used in conjunction with stochastic finite elements in order to capture deterministic and statistic size effects simultaneously.

In this paper, we will employ a nonlocal damage continuum of the differential format proposed in [14] and a viscoplastic damage continuum as described in [15]. Three-point bending specimens are analysed numerically by means of the finite element method, and a distinction is made between three types of beams: unnotched beams, notched beams where the notch dimensions do not scale with the specimen size (referred to as "constant notch") and notched beams where the notch dimensions scale proportionally with the other dimensions of the specimen (referred to as "proportional notch"). Whereas the cases of unnotched beams and beams with a proportional notch have been covered extensively in the literature and used as validation for the above-mentioned size effect models, beams with a constant notch may be more relevant for engineering practice: when notches are supposed to represent defects in the material, the notch dimensions are set by the material length scale and not by the structural length scale.

\section{Constitutive relationships}

Common to the two classes of regularised constitutive models considered in this contribution is the use of the damage framework to represent void development. The stress-strain relation is written as

$$
\boldsymbol{\sigma}=(1-\omega) \boldsymbol{C} \varepsilon
$$

where $\sigma$ and $\varepsilon$ contain the components of stresses and strains, $\boldsymbol{C}$ contains the elastic moduli and $\omega$ is a scalar damage variable. To avoid mesh dependence, damage evolution must be postulated as some function of a regularised monotonically increasing deformation history invariant $\kappa$. For the gradient-enhanced continuum damage model, damage evolution is made a function of the nonlocal equivalent strain, while in the rate-dependent elastoplastic damage model, damage is made a function of the equivalent viscoplastic strain. The two 
frameworks differ significantly in the nature of the regularisation involved (temporal regularisation versus spatial regularisation) and in the dissipation mechanism. It is noted that in the rate-dependent elastoplastic damage model, damage is plastically-induced.

In the gradient-enhanced continuum damage model, an equivalent strain is defined according to the Modified Von Mises criterion as

$\varepsilon_{\mathrm{eq}}=\frac{k-1}{2 k(1-2 v)} I_{1}+\frac{1}{2 k} \sqrt{\frac{(k-1)^{2}}{(1-2 v)^{2}} I_{1}^{2}+\frac{6 k}{(1+v)^{2}} J_{2}}$,

where $I_{1}$ and $J_{2}$ are the first and second invariant of the strain tensor and the deviatoric strain tensor, respectively, and $k$ denotes the compressive-to-tensile strength ratio. The local equivalent strain $\varepsilon_{\text {eq }}$ defined in Equation (2) is translated into its nonlocal counterpart $\bar{\varepsilon}_{\text {eq }}$ via a Helmholtz-type relation as

$$
\bar{\varepsilon}_{\mathrm{eq}}-\frac{1}{2} l^{2} \nabla^{2} \bar{\varepsilon}_{\mathrm{eq}}=\varepsilon_{\mathrm{eq}},
$$

where $l$ is a material length parameter that represents the underlying microstructure. Equation (3) is augmented by Neumann boundary conditions throughout and solved simultaneously with the equilibrium equations that involve Equation (1), thus leading to a coupled system of equations, see for instance [14].

As usual, a history variable $\kappa$ is introduced as

$$
\kappa=\max \left(\kappa_{i}, \bar{\varepsilon}_{\mathrm{eq}}\right),
$$

where $\kappa_{i}$ represents the crack initiation strain. Upon loading, damage grows according to

$$
\omega=1-\frac{\kappa_{i}}{\kappa} \exp \left(-\beta\left(\kappa-\kappa_{i}\right)\right) \text { if } \kappa>\kappa_{i}
$$

in which $\beta$ is a material parameter that sets the slope of the stress-strain relation in the softening regime.

The differential format of nonlocal damage as described above can equally be written in an integral form, whereby the weight function takes the format of a Green's function [16]. Indeed, the differences between the differential format and the integral format of nonlocal damage are merely quantitative $[17,18,16]$.

In the rate-dependent elastoplastic damage model, damage evolution is postulated as [15]

$$
\omega=\alpha(1-\exp (-\beta \kappa))
$$

with $\alpha$ and $\beta$ model parameters and $\kappa_{i}$ the threshold of damage initiation. The viscoplastic strain rate is expressed, in presence of plastic flow ( $f \geq 0$, where $f$ is the yield function), in the associative form [19]

$$
\dot{\varepsilon}^{\mathrm{vp}}=\frac{1}{\tau} \phi \partial_{\sigma} f,
$$

where the overstress function is given the following power-law form:

$$
\phi(f)=\left(\frac{f}{\bar{\sigma}_{0}}\right)^{N},
$$

with $\bar{\sigma}_{0}$ the initial yield stress and $N(N \geq 1)$ a real number. The yield stress has been given an exponential form according to

$$
\bar{\sigma}(\kappa)=\bar{\sigma}_{0}((1+a) \exp (-b \kappa)-a \exp (-2 b \kappa))
$$

with $a$ and $b$ model parameters and $\bar{\sigma}_{0}$ the initial cohesion (or yield stress).

The rate-dependent isotropic elastoplastic damage model is discussed in detail in [15] where the algorithmic treatment is presented and the regularisation properties are illustrated. Details of the implicit gradient-enhanced continuum damage model are discussed in [14].

\section{Description of numerical simulations}

For the size effect analysis three-point bending tests are simulated.

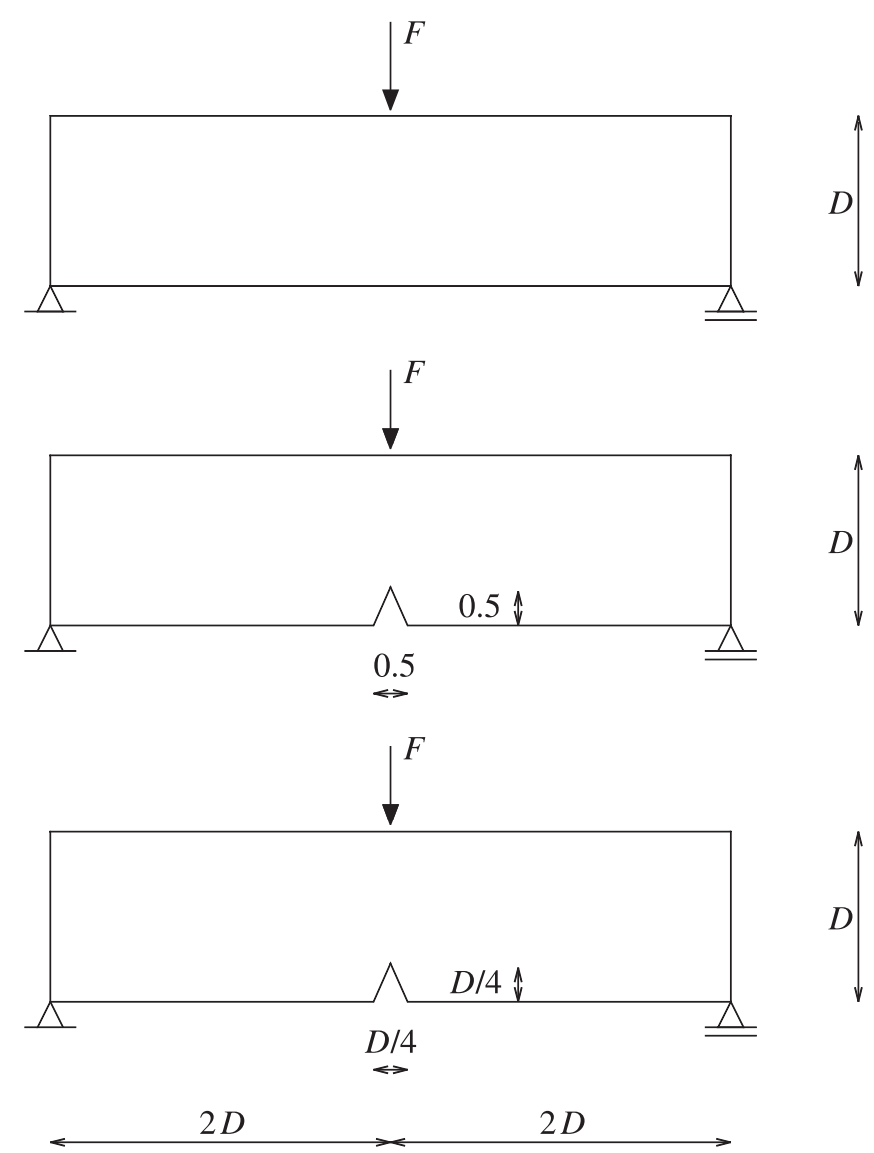

Fig. 1: Geometries of three-point bending specimens: unnotched beam (top), beam with constant notch (middle) and beam with proportional notch (bottom)

Fig. 1 shows the three sets of geometries that are studied, namely

- Unnotched beams: The dimensions of the beam are simply taking as span $\times$ height $=4 D \times D$ and no notch is present.

- Beams with a constant notch: The outer dimensions of the beam are the same as for the unnotched case, but a wedge-shaped notch is assumed with dimensions base $\times$ height $=0.5 \mathrm{~mm} \times 0.5 \mathrm{~mm}$. The dimensions of the notch do not scale with the dimensions of the beam. 
- Beams with a proportional notch: Again, the outer dimensions of the beam are taken similar to the unnotched case. Wedge-shaped notches are assumed that scale proportionally with the beam dimensions: for the notch we take base $\times$ height $=0.25 D \times 0.25 D$.

For all three cases we study size ranges of $D=1 \mathrm{~mm}$ up to $D=64 \mathrm{~mm}$. The constant notch case and the proportional notch case coincide for $D=2 \mathrm{~mm}$.

The elastic constants used in the nonlocal damage continuum are $E=30000 \mathrm{MPa}$ and $v=0.15$ while plane strain conditions are assumed. For the damage evolution we use $\kappa_{\mathrm{i}}=0.0001$ and $\beta=500$; the compressive-to-tensile strength ratio is taken as $k=10$. The material length scale in all analyses equals $l=1 \mathrm{~mm}$. This implies that for the smallest specimens $(D=1 \mathrm{~mm})$ the structural dimensions and the material length are in the same order of magnitude, whereas for the largest specimens $(D=64 \mathrm{~mm})$ the material length scale is negligibly small compared to the structural dimensions. In the viscoplastic damage continuum we have used the same elastic constants of the nonlocal damage continuum and a plane stress smoothed Rankine yield function with $\bar{\sigma}_{0}=f_{t}=2 \mathrm{MPa}$ and with softening parameters $a=-1$, $b=200, \alpha=0.999$ and $\beta=5000$. The exponent of the overstress function is $N=1$ and the relaxation time is $\tau=10 \mathrm{~s}$.

For the numerical analyses we have used finite element meshes consisting of three-node triangles with linear shape functions for the displacements as well as for the nonlocal equivalent strain [20]. The mesh density is determined by two factors: in the central section of the beams, a sufficiently fine mesh must be used to capture damage initiation and damage propagation accurately; moreover, a sufficient number of elements over the beam height must be used to describe the bending behaviour without locking effects. Representative element sizes of $0.25 \mathrm{~mm}$ have been employed in the centre of the beams, while it has been ensured that at least 5 elements over the beam height are present.

\section{Results}

Size effects are investigated in terms of nominal strength, denoted as $\sigma$, which is defined as peak load divided by $D \times 1 \mathrm{~mm}^{2}$. In Figs. 2 and 3 the nominal strength is plotted as a function of the structural dimension $D$ in the usual logarithmic scale. It can be seen that for both regularised continuum formulations and for all three geometries a size effect in nominal strength is obtained. Furthermore, this size effect persists in the whole size range. For larger specimen sizes, the unnotched specimens and the specimens with a constant (non-proportional) notch behave similarly in that the same slope is obtained in the size effect curves. However, the presence of a notch lowers the nominal strength, even if the notch dimensions are negligible compared to the structural dimensions. The beams with a proportionally scaled notch behave significantly different from the other two test series: a steeper (i.e. more negative) slope in the size effect diagrams is obtained.

The two regularised continua follow the same trends, apart from the extreme sensitivity of the constant notch beam with small dimensions. In the nonlocal damage formulation

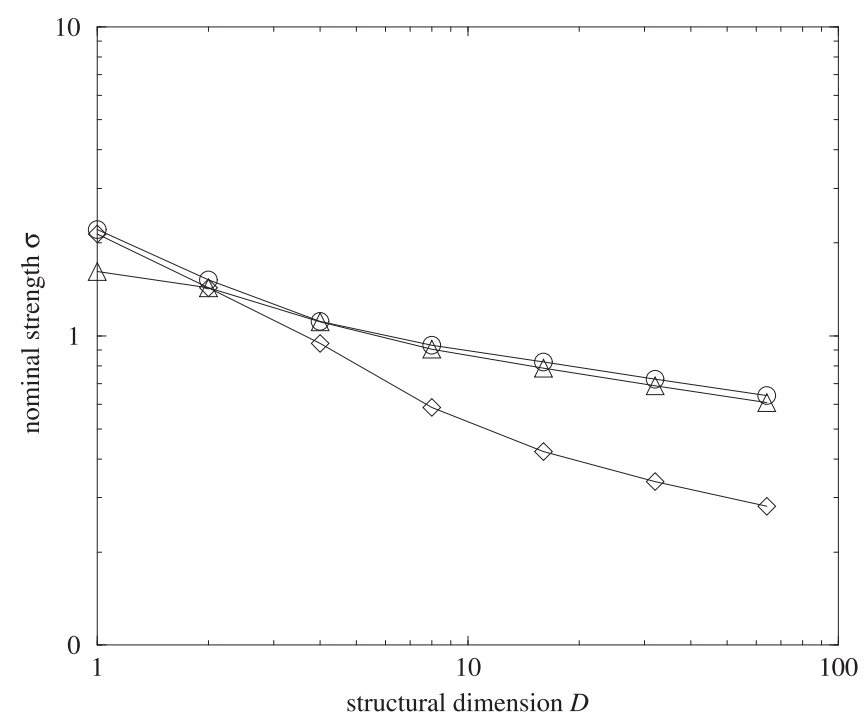

Fig. 2: Nonlocal damage: size effects for unnotched beams (circles), beams with constant notch (triangles) and beams with proportional notch (diamonds)

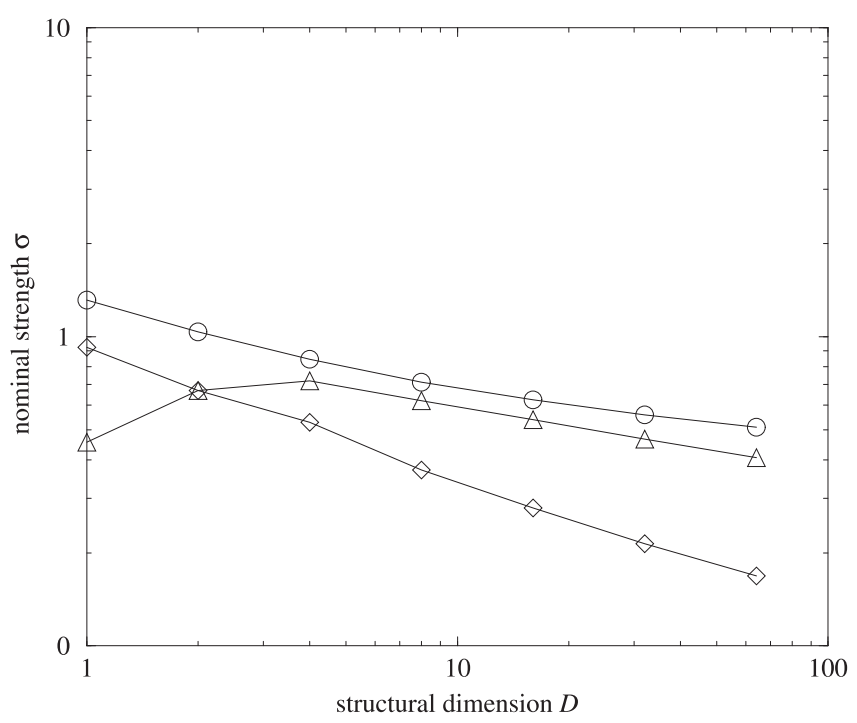

Fig. 3: Viscoplastic damage: size effects for unnotched beams (circles), beams with constant notch (triangles) and beams with proportional notch (diamonds)

the constant notch case does not differ very much from the other two cases for small specimen sizes, whereas in the viscoplastic damage formulation a more significant difference exists. The small size range implies that the notch dimension is in the same order of magnitude as the material length scale. As is argued in [21], the imperfection (here: notch) dominates the response in a viscosity-enhanced continuum when the material length scale and the notch dimensions are in the same order of magnitude, while a nonlocal continuum is much less sensitive to notch dimensions.

For theoretical predictions it is relevant to validate the obtained numerical results with the proposed size effect models of the literature. To this end, we have fitted the numerical results of the unnotched beams with the Multi-Fractal Scaling Law and the numerical results of the proportion- 


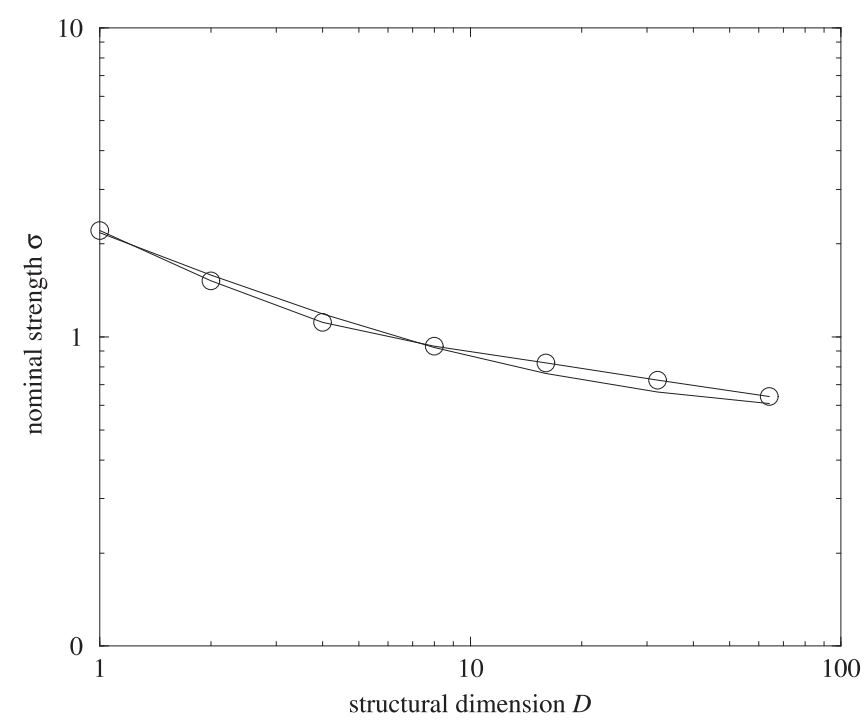

Fig. 4: Nonlocal damage: size effects for unnotched beams: numerical experiments (solid with circles) versus Multi-Fractal Scaling Law (dashed)

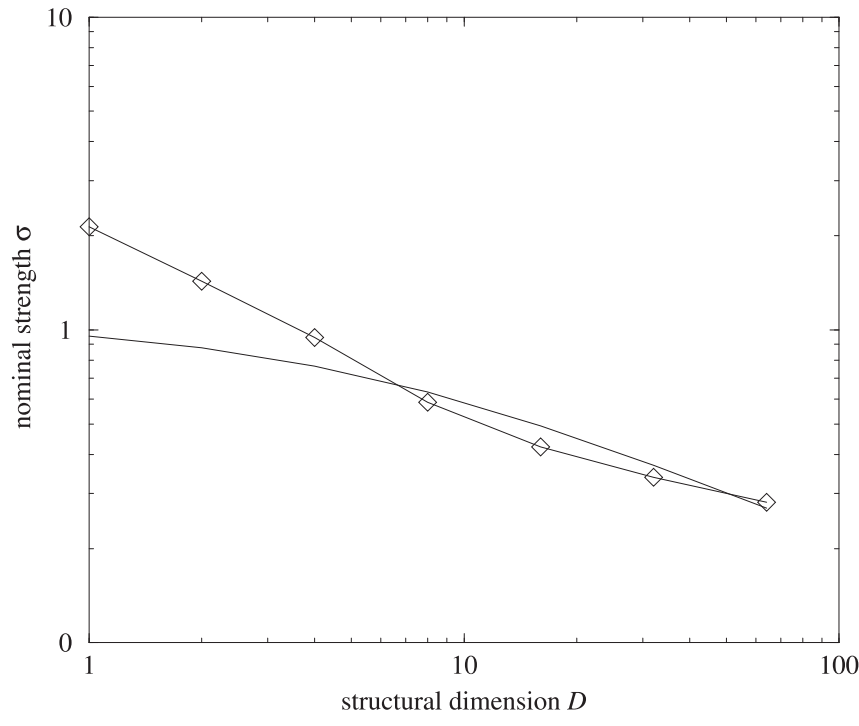

Fig. 5: Nonlocal damage: size effects for proportionally notched beams: numerical experiments (solid with diamonds) versus Size Effect Law (dashed)
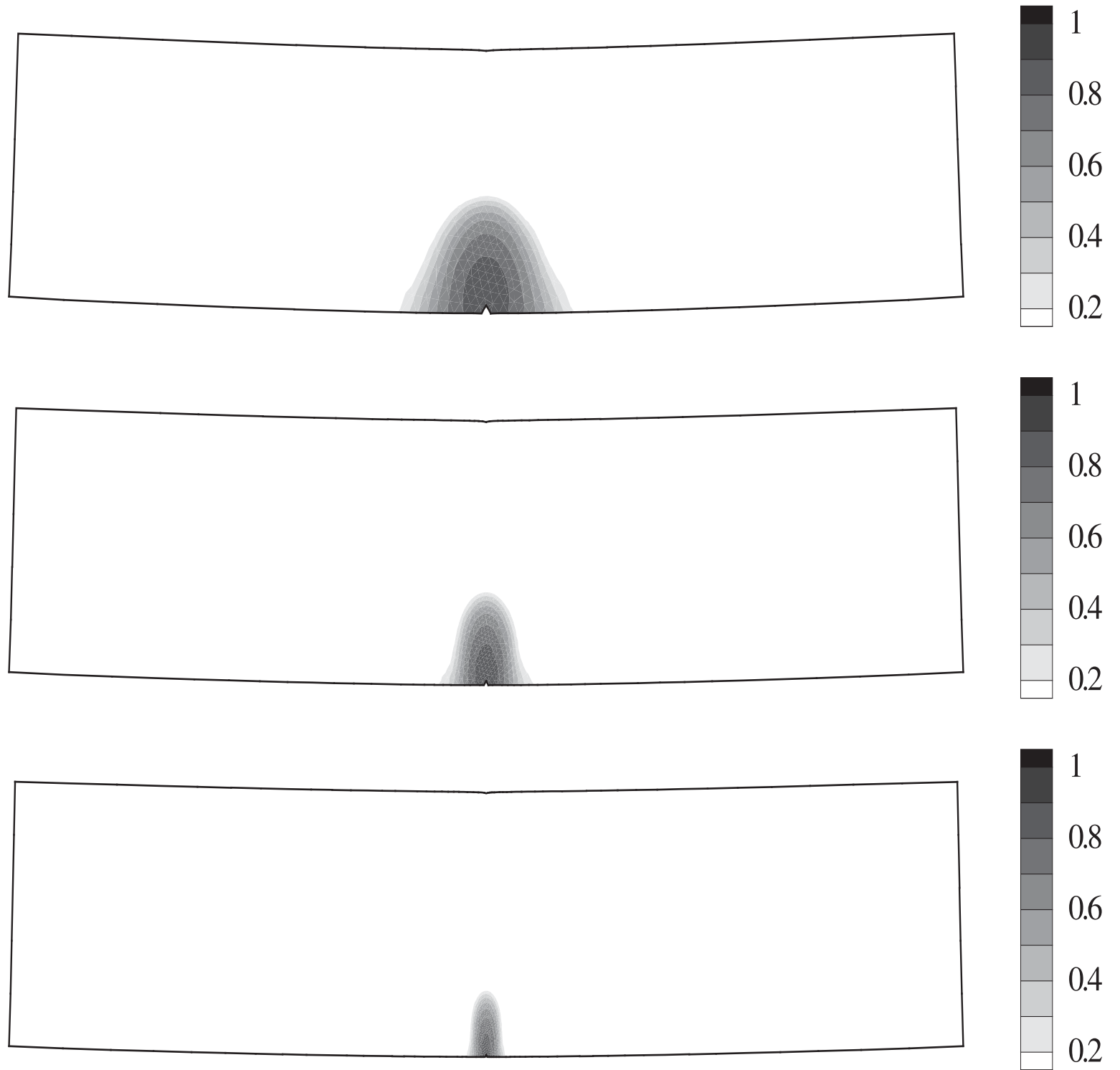

Fig. 6: Damage contours at peak load in beams with constant notch: $D=16 \mathrm{~mm}$ (top), $D=32 \mathrm{~mm}$ (middle) and $D=64 \mathrm{~mm}$ (bottom). Deformed configurations are plotted with magnification factor 100 

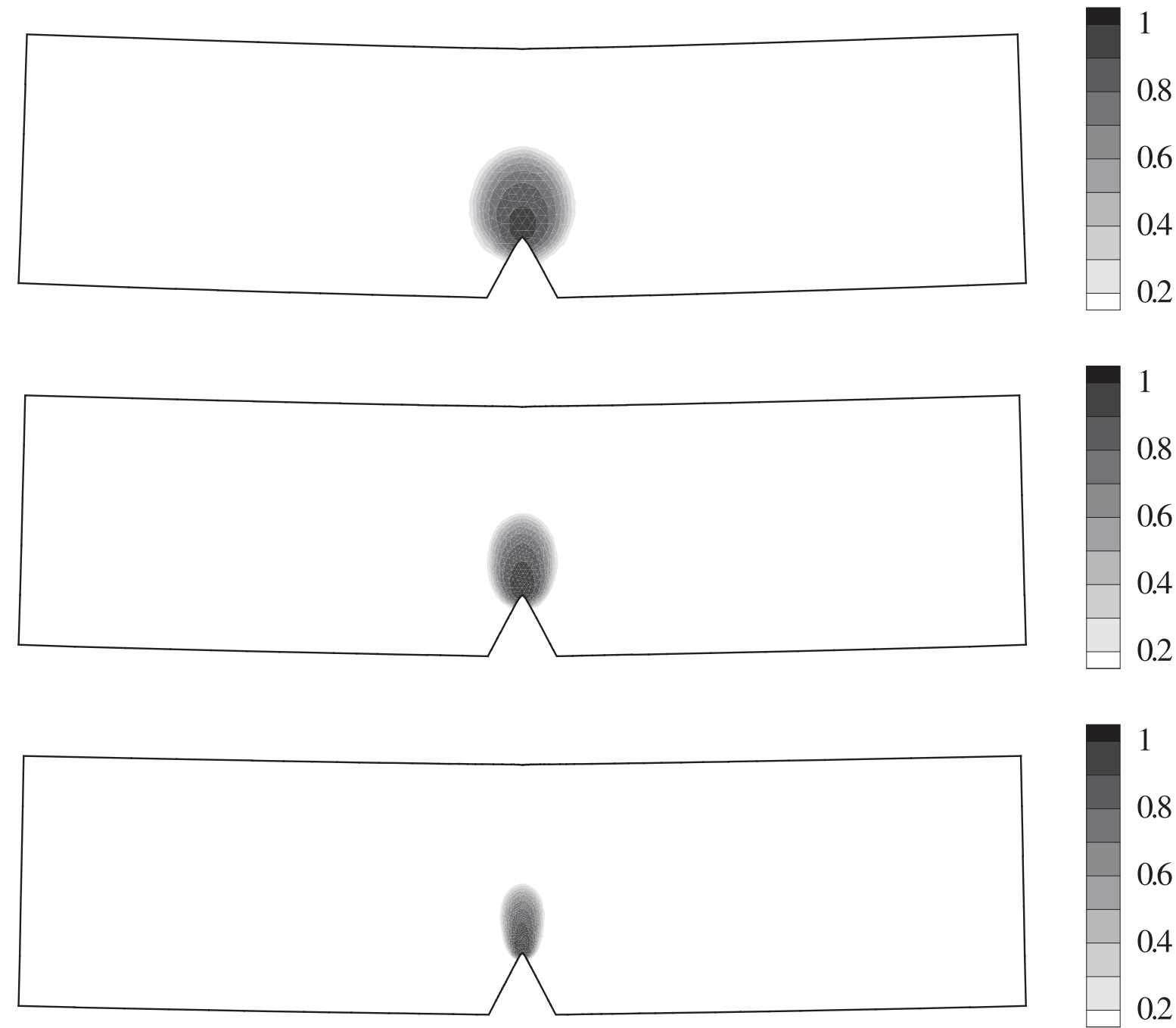

Fig. 7: Damage contours at peak load in beams with proportional notch: $D=16 \mathrm{~mm}$ (top), $D=32 \mathrm{~mm}$ (middle) and $D=64 \mathrm{~mm}$ (bottom). Deformed configurations are plotted with magnification factor 100

ally notched beams with the Size Effect Law, see Figs. 4 and 5 , using the nonlocal damage formulation. A least-squares fitting procedure has been used. With the current set of material and geometrical parameters, the size effect models quantify as

$$
\sigma=0.5482 \sqrt{1+\frac{14.7492}{D}}
$$

for the Multi-Fractal Scaling Law, and

$$
\sigma=\frac{1}{\sqrt{1-\frac{D}{4.4640}}}
$$

for the Size Effect Law.

As can be seen in Fig. 4, the Multi-Fractal Scaling Law provides a reasonable prediction of the numerically obtained results, although a finite slope in the size effect diagram is found numerically that cannot be recovered by means of the Multi-Fractal Scaling Law (which approaches a horizontal plateau in the large-size range). This can be understood when studying the damage contours at the peak load, for instance of the beams with a constant notch, as are displayed for $D=16 \mathrm{~mm}, D=32 \mathrm{~mm}$ and $D=64 \mathrm{~mm}$ in Fig. 6. A horizontal slope in the size effect curve would imply that the uncracked portion of the beam height is proportional to the structural size $D$, thereby assuming that the same distributions of damage and stress will be reached along the vertical symmetry axis. However, from Fig. 6 it is seen that this is clearly not the case if the present nonlocal damage continuum is used: the uncracked portion of the beam decreases as structural dimension $D$ increases.

More severe deviations are found for the proportionally notched beams, cf. Fig. 5: the numerical results exhibit a convexity in the large-size range where the Size Effect Law is strictly concave. For the large-size range, the Size Effect Law predicts a slope of the size effect curve of 1:2 in a log-log scale, while the numerical results tend to converge towards a much less steep slope. For the large-size range, the material model that underlies the Size Effect Law is Linear Elastic Fracture Mechanics (LEFM). Compared to LEFM, a nonlocal contin- 
uum has an increased nominal strength due to the material length scale, which is lacking in LEFM. Also, singularities are removed in the present nonlocal continuum [16, 22] which leads to an increased nominal strength compared to LEFM. In the small-size range large differences are obtained between the numerically obtained size effect and the predictions via the Size Effect Law. However, following the argumentation outlined in the Introduction, these differences are considered to be of minor relevance. In Fig. 7 the damage contours at the peak load are plotted for $D=16 \mathrm{~mm}, D=32 \mathrm{~mm}$ and $D=64 \mathrm{~mm}$. The damaged region has extended significantly, even for the largest size, so that the ductility is significantly increased.

\section{Closure}

Size effects are studied from a numerical point of view. A nonlocal damage continuum of the differential type and a viscoplastic damage continuum have been employed, both of which include a material length scale. Three-point bending tests have been analysed with and without notches, where notches were considered that do or do not scale with the structural dimensions (proportional notches and constant notches, respectively).

The size effects exhibited by unnotched beams and by beams with a constant notch are virtually identical in the large-size range. The numerically obtained results differ somewhat from the Multi-Fractal Scaling Law, which is due to the different mechanics that underlies the nonlocal continuum. The size effects shown by proportionally notched beams are in reasonable agreement with the Size Effect Law. The difference between the two types of regularisation (spatial gradients versus viscosity) is negligible for the size-effect predictions. It is emphasised that the slopes of the numerically obtained size effects in the usual log-log diagrams are bounded by the Multi-Fractal Scaling Law from above and by the Size Effect Law from below. Using the two regularised continua discussed in this paper, neither the Multi-Fractal Scaling Law nor the Size Effect Law is obtained in the large-size range.

\section{References}

[1] Carpinteri A. (editor): "Size-scale effects in the failure mechanisms of materials and structures." E. \& F.N. Spon, London, 1996.

[2] Aifantis E.C.: "Strain gradient interpretation of size effects." International Journal of Fracture, Vol. 95 (1999), p. 299-314.

[3] Bažant Z. P.: "Size effect." International Journal of Solids and Structures, Vol. 37 (2000), p. 69-80.

[4] Karihaloo B. L., Xiao Q. Z.: "Size effect in the strength of concrete structures.” Sadhana, Vol. 27 (2002), p. $449-459$.

[5] van Mier J. G. M., van Vliet M. R. A.: "Influence of microstructure of concrete on size/scale effects in tensile fracture." Engineering Fracture Mechanics, Vol. 70 (2003) p. 2281-2306.
[6] Fleck N. A., Muller G. M., Ashby M. F., Hutchinson J. W.: "Strain gradient plasticity: theory and experiment." Acta Metallurgica et Materialia, Vol. 42 (1994), p.475-487.

[7] Zhu H. T., Zbib H. M., Aifantis E. C.: "Strain gradients and continuum modeling of size effect in metal matrix composites." Acta Mechanica, Vol. 121 (1997), p. 165-176.

[8] Efremidis G., Carpinteri A., Aifantis E. C.: “Griffith's theory versus gradient elasticity in the evaluation of porous materials tensile strength." Journal of the Mechanical Behavior of Materials, Vol. 12 (2001), p. 95-105.

[9] Efremidis G., Carpinteri A., Aifantis E. C.: "Multifractal scaling law versus gradient elasticity in the evaluation of disordered materials compresive strength." Journal of the Mechanical Behavior of Materials, Vol. 12 (2001), p. 107-120.

[10] Askes H., Aifantis E. C.: "Numerical modeling of size effects with gradient elasticity - Formulation, meshless and examples." International Journal of Fracture, Vol. 117 (2002), p. 347-358.

[11] Carmeliet J.: "Optimal estimation of gradient damage parameters from localization phenomena in quasi-brittle materials." Mechanics of Cohesive-Frictional Materials, Vol. 4 (1999), p. 1-16.

[12] le Bellégo C., Dubé J. F., Pijaudier-Cabot G., Gérard B.: "Calibration of nonlocal damage model from size effect tests." European Journal of Mechanics A/Solids, Vol. 22 (2003), p. 33-46.

[13] Gutiérrez M. A., de Borst R.: "Deterministic and stochastic analysis of size effects and damage evolution in quasi-brittle materials." Archive of Applied Mechanics, Vol. 69 (1999), p. 655-676.

[14] Peerlings R. H. J., de Borst R., Brekelmans W. A. M., de Vree J. H. P.: "Gradient enhanced damage for quasi-brittle materials." International Journal for Numerical Methods in Engineering, Vol. 39 (1996), p. 3391-3403.

[15] Simone A.,. Sluys L. J.: "The use of displacement discontinuities in a rate-dependent medium." Computer Methods in Applied Mechanics and Engineering, Vol. 193 (2004), p. 3015-3033.

[16] Peerlings R. H. J., Geers M. G. D., de Borst R., Brekelmans W. A. M.: "A critical comparison of nonlocal and gradient-enhanced softening continua." International Journal of Solids and Structures, Vol. 38 (2001), p. 7723-7746.

[17] Peerlings R. H. J., de Borst R.,. Brekelmans W. A. M, de Vree J. H. P., Spee I.: "Some observations on localisation in non-local and gradient damage models." European Journal of Mechanics, A/Solids, Vol. 15 (1996), p. 937-953.

[18] Askes H., Pamin J., de Borst R.: "Dispersion analysis and Element-Free Galerkin solutions of second and fourth-order gradient-enhanced damage models." International Journal for Numerical Methods in Engineering, Vol. 49 (2000), p. 811-832.

[19] Perzyna P.: "Fundamental problems in viscoplasticity." Advances in Applied Mechanics, Vol. 9 (1966), Academic Press, New York, p. 243-377. 
[20] Simone A., Askes H., Peerlings R. H. J., Sluys L. J.: "Interpolation requirements for implicit gradient-enhanced continuum damage models." Communications in Numerical Methods in Engineering, Vol. 19 (2003), p. 563-572. (See also the corrigenda: Communications in Numerical Methods in Engineering, Vol. 20 (2004), p. 163-165.)

[21] Wang W. M., Sluys L. J., de Borst R.: "Interaction between material length scale and imperfection size for localisation phenomena in viscoplastic media." European Journal of Mechanics A/Solids, Vol. 15 (1996), p. 447-464.

[22] Simone A., Askes H., Sluys L. J.: "Incorrect initiation and propagation of failure in non-local and gradient-enhanced media." International Journal of Solids and Structures, Vol. 41 (2004), p. 351-363.

\section{H. Askes}

Faculty of Civil Engineering and Geosciences Delft University of Technology

PO Box 5048

NL-2600 GA Delft, Netherlands

\section{A. Simone}

Faculty of Mathematics and Natural Sciences University of Groningen

Nijenborgh 4

NL-9747 AG Groningen, Netherlands

\section{J. Sluys}

Faculty of Civil Engineering and Geosciences Delft University of Technology

PO Box 5048

NL-2600 GA Delft, Netherlands 